\title{
Approaches to modeling operational risks of frequency and severity in insurance
}

\author{
Fatima Zahra El Arif ${ }^{1}$, Nadia Lamchichi ${ }^{2}$, Amine Dafir ${ }^{3}$ \\ ${ }^{I}$ PhD student - Center of Doctoral Studies, Faculty of Economic Sciences, University Mohammed V-Souissi, \\ Rabat, Morocco. \\ ${ }^{2}$ PhD student - Center of Doctoral Studies, Faculty of Economic Sciences, University Mohammed V-Souissi, \\ Rabat, Morocco. \\ ${ }^{3}$ PhD - Center of Doctoral Studies, Faculty of Economic Sciences, University Mohammed V-Souissi, Rabat, \\ Morocco.
}

\begin{abstract}
One of the great new of Solvency II is the requirement for insurance companies to raise some of their own funds to cover their exposure to operational risks. To calculate this amount of capital, the regulator proposes two approaches : a standard approach and an advanced approach. The standard approach is a simplified approach calculated as a percentage of premiums or reserves. The advanced approach is an internal model where the risk actually corresponds to the situation of the company. EIOPA encourages insurance companies to opt for internal model by making the standard approach much more consuming in stockholders' equity. Thus, we propose in this paper a modeling by advanced approach that distinguishes frequency risks of severity risks. Frequency risks are defined as potential loss of low but frequent amounts : they are modeled by the Loss Distribution Approach. Severity risks are risks of losses of very important but very few amounts : they are modeled by Bayesian networks.
\end{abstract}

Keywords: Operational risk, standard approach, advanced approach, frequency risks, severity risks, Loss Distribution Approach, Bayesian networks, insurance.

\section{Introduction}

The definition of operational risk is a challenge. This risk has an atypical character as far as it concerns all the activities of the insurance. It is also often difficult to estimate it and quantify it independently of other risks which characterizes the activity of insurance.

Indeed, the management practices and measure of this risk are based very heavily on what happens on the market risk, the maturity of which is much more important. However, approaches (such as Loss Distribution Approach or LDA, and Bayesian networks) are becoming increasingly important to manage and quantify this risk, especially since the establishment of the systems of collection of losses.

To identify the methodology to be used, the type of risk is identified during the risk mapping and should be reviewed periodically.

Frequency risks are defined as potential loss of low but frequent amounts : they are modeled by the Loss Distribution Approach. Severity risks are risks of losses of very important but very few amounts : they are modeled by Bayesian networks.

However, the risks of important and frequent losses will not be modeled, because when a severity risk arises, the insurance company will establish checks and other action plans, that will reduce or eliminate this risk (black box) [Fig. 1].

\section{Modeling Of Frequency Risk : Loss Distribution Approach}

Loss Distribution Approach is to adjust statistical laws to data loss, specifically modeled on the one hand, the frequency of operational incidents, and secondly, their severity, and then combine them to obtain the distribution of total losses. This approach is often used to model the total cost of claims in the context of pricing and provisioning. The essential condition for applying this method will be the availability of historical losses data to calibrate the model.

II.1. Step 1: selection and calibrating of frequency and severity laws Modeling the distribution of losses will for each risk $\mathrm{k}$ :

$$
S_{k}=\sum_{j=1}^{N_{k}} X_{k}^{(j)}
$$

Where $\mathrm{N}_{\mathrm{k}}$ is the random variable representing the number of losses for risk $\mathrm{K}$, 
$\mathrm{X}_{\mathrm{k}}^{(\mathrm{j})}$ is the random variable representing the cost of the loss $\mathrm{j}$ for risk $\mathrm{k}, \mathrm{S}_{\mathrm{k}}$ is the sum of the losses of risk $\mathrm{k}$.

The main laws used for frequency are usually Poisson, Binomial and Negative Binomial.

There is a near consensus place to use the method of maximum likelihood to estimate the parameters of the law. The choice of model is then validated by statistical tests.

\section{II.2. Step 2 : construction of total losses distribution}

It is possible to obtain a good approximation of the distribution function of the distribution of losses $S_{k}$, with the method of Monte-Carlo simulation [Fig. 2].

\section{II.2.1. Practical problem : the collection threshold}

Generally, operational will not report all losses: it will then establish a reporting threshold. The observations which we will have will be losses that exceed this threshold. It will thus take into account this truncation in our adjustments and changes in estimates of parameters and fit testing accordingly.

Indeed, if we do not take into account the fact that the data is truncated, we will underestimate the frequency, and adjust the law of the individual costs incorrectly.

\section{II.2.2. Law of individual cost}

The data below the threshold are not ski, but must be taken into account in the estimation of parameters : it will estimate the parameters of the law taking into account the lack of data on the left of the histogram as we watch in the graphic [Fig. 3].

Let $\mathrm{U}$ be the collection threshold losses.

Let $\mathrm{X}$ be the random variable representing the cost of non-truncated losses (that is to say losses, the amount may be less than $\mathrm{U}$ ) and $\mathrm{F}$ its distribution function.

This conditional law is the law of $\mathrm{X}$ truncated to the left of $\mathrm{U}$.

We can explain the distribution function of the truncated law knowing that the observations are beyond the threshold U :

For all $\mathrm{x}>\mathrm{U}$

$$
P(X<x \mid X \geq U)=\frac{P(U \leq X<x)}{P(X \geq U)}=\frac{F(x)-F(U)}{1-F(U)}
$$

For all $\mathrm{x} \leq \mathrm{U}$

$$
\mathrm{P}(\mathrm{X}<\mathrm{x} \mid \mathrm{X} \geq \mathrm{U})=0
$$

The graph [Fig. 4] shows the deformation of a log-normal law $\ln (2,1)$, depending on the level of collection threshold ${ }^{1}$.

Let $\left(\mathrm{x}_{1}, \mathrm{x}_{2}, \ldots, \mathrm{x}_{\mathrm{n}}\right)$ a sample of losses that exceed the threshold $\mathrm{U}$, the conditional likelihood in threshold $\mathrm{U}$ can be written :

$$
\prod_{i=1}^{n} \frac{f\left(x_{i}\right)}{P\left(X_{i} \geq U\right)}=\prod_{i=1}^{n} \frac{f\left(x_{i}\right)}{1-F(U)}
$$

The log-likelihood can be then written :

$$
\sum_{i=1}^{n} \ln \left(\frac{f\left(x_{i}\right)}{1-F(U)}\right)=\sum_{i=1}^{n} \ln \left(f\left(x_{i}\right)\right)-n \ln (1-F(U))
$$

The parameters are estimated in a conventional manner by maximizing the log-likelihood.

\section{II.2.3. Law of frequency}

Where there is a collection threshold, the frequency of observed losses is underestimated and must be adjusted to account for unreported losses.

For each observation period $i$, the number of claims noted $n_{i}$ is increased by the estimated number of claims below the threshold noted $\mathrm{m}_{\mathrm{i}}$ :

$$
\mathrm{n}_{\mathrm{i}}^{\mathrm{r}}=\mathrm{n}_{\mathrm{i}}+\mathrm{m}_{\mathrm{i}}
$$

\footnotetext{
${ }^{1}$ Note : Some distributions are stable by a truncation to the left as the Exponential and Pareto laws. The method of maximum likelihood allows us to calibrate our laws, but does not give us an analytical solution.
} 
The ratio between the number of losses below the threshold and the number of losses observed, is the same as the ratio between the area below the curve of the density of the individual cost situated to the left of the threshold, and that was situated to the right of the threshold :

$$
\frac{m_{i}}{n_{i}}=\frac{\widehat{F}(U)}{1-\hat{F}(U)}
$$

Where $\mathrm{F}$ is the function of the truncated distribution of $\mathrm{X}$, whose parameters have been estimated with the previous method.

From where :

$$
n_{i}^{r}=n_{i}+m_{i}=n_{i}+\frac{n_{i \times \widehat{F}(U)}}{1-\widehat{F}(U)}=\frac{n_{i}}{1-\widehat{F}(U)}
$$

\section{Modeling Of Severity Risk : Bayesian Method}

The Bayesian approach is to conduct a qualitative risk analysis by experts and turn it into a quantitative analysis.

A Bayesian network is a probabilistic causal graph representing the structure of knowledge in a certain field. It consists of discrete random variables connected by directed arcs, these variables are called nodes. Distribution is attached to each node. Arcs are links that represent causal dependence.

We studied in particular the method XOS (exposure, occurrence, severity), which is to define and model the three characteristic parameters of risk : the exposure, the occurrence and the severity. These three variables are influenced by variables called Key Risk Indicator (KRI).

We are going to present the methods to estimate the various elements of the Bayesian network.

- Assessment of exposure : The exposure is all the elements of the company which are exposed to the risk. She must be defined so that the risk can arise only once in most in the year.

- $\quad$ Assessment of occurrence : The item of exposition being selected so that it can be stukch only in most only once, the occurrence will be built by a binomial distribution $B(n, p)$, where $n$ is the number of exhibits and $\mathrm{p}$ the probability which we shall have to estimate.

- $\quad$ Assessment of severity : It is necessary to take place in the situation where the occurrence of the loss is proven, and to identify the quantifiable variables (KRI) occuring in the calculation of the gravity.

The structure of the Bayesian network is defined by the experts through scenarios. Bayesian network parameters can be determined empirically or by experts.

Once the Bayesian network built, it remains to define the algorithm of calculation.

Let $\left(\mathrm{X}_{1}, \mathrm{X}_{2}, \ldots, \mathrm{X}_{\mathrm{n}}\right)$ are exposed objects at the studied operational risk.

Let $\mathrm{P}_{\mathrm{i}}=\mathrm{P}\left(\right.$ Exposure $\left.=\mathrm{X}_{\mathrm{i}}\right)$ the probability that exposure let be objects $\mathrm{X}_{\mathrm{i}}$.

Let $\mathrm{PS}_{\mathrm{i}}=\mathrm{P}\left(\right.$ Occurrence $=$ "yes" $\mid$ Exposure $\left.=\mathrm{X}_{\mathrm{i}}\right)$ the probability of the risk occurring given that the exposure is $\mathrm{X}_{\mathrm{i}}$.

These two probabilities are known (they were estimated as seen above).

Let $\mathrm{PG}_{\mathrm{i}}$ is $=\mathrm{P}\left(\right.$ Severity $\mid$ Occurrence $=$ "yes" and Exposure $\left.=\mathrm{X}_{\mathrm{i}}\right)$ the distribution of the severity knowing that the risk occurred on $\mathrm{X}_{\mathrm{i}}$ objects.

The algorithm consists in realizing successively the following steps :

1. position exposure to $X_{i}$, the occurrence to Yes in the Bayesian network and read the distribution of severity $\mathrm{PG}_{\mathrm{i}}=\mathrm{P}\left(\right.$ Severity $\mid$ Occurrence $=$ "yes" and Exposure $\left.=\mathrm{X}_{\mathrm{i}}\right)$.

2. sample the number of losses $F_{i}$ according to binomial law $B\left(n b\left(X_{i}\right) ; P_{i}\right)$.

3. for each incident of 1 to $F_{i}$, sample the severity according to the $P_{G_{i}}$ distribution.

4. sum the severities $F_{i}$.

By repeating these 4 steps a large number of time by keeping the sums of the severity every time, we so obtain a distribution of total losses.

\section{Example Of Application Of The Bayesian Method}

We now apply the Bayesian model to the risk of error in the passage of orders, on an insurance company. This risk is treated very simply because our idea is to show how the Bayesian approach applies.

The first step in the Bayesian approach is to create the network using a graph, and define the distributions corresponding to each factor. These parameters can be reviewed in a phase of "back-testing". An analysis of this risk has allowed us to build the graph [Fig. 5].

Recall that the Bayesian approach proposed for operational risk is to define three objects : the exposure, the occurrence and the severity ${ }^{2}$.

\footnotetext{
${ }^{2}$ The XOS method we described above.
} 
The exposure : the exposure has to correspond to the objects of exposition of the company which can be touched by the risk only once for the period.

We chose the orders. Indeed, an order can be erroneous only once. We do not anticipate increase in the number of orders for year to come and the number of observed orders is on average $25 \mathrm{OOO}$ a year.

The occurrence : The occurrence is defined as the error on an order. On average, we have a number of annual losses of 18.2, is a probability of error on the order of $18.2 / 25000=0.0728 \%$.

The severity : severity is defined as the sum of the loss due to shifting and transaction costs. We make the following assumptions about the factors involved in the calculation of the severity.

- The amount of the error (expressed in millions of euros) :

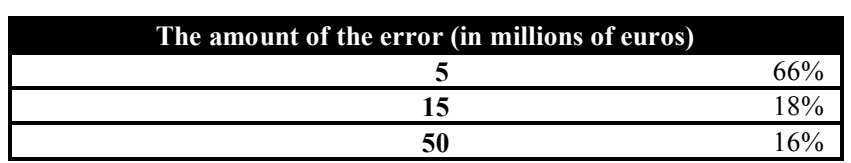

- The duration of error correction : the period from the date of occurrence of the error to the date of correction by the passage of a new order :

\begin{tabular}{|rr|}
\hline \multicolumn{2}{|c|}{ The duration of correction (in days) } \\
\hline $\mathbf{0 , 1 2 5}$ & $66 \%$ \\
\hline $\mathbf{1}$ & $33 \%$ \\
\hline $\mathbf{9 0}$ & $1 \%$ \\
\hline
\end{tabular}

- The transaction costs : we assume that transaction costs are equal to $0.25 \%$ of the transaction amount. The amount of error is passed first time and a second time during the correction. The fees will be calculated by multiplying the amount of the error by $0.5 \%$.

- The rate shifts : it is about the variation of the rates observed during the duration of correction of the error.

- The loss due to shift : it is calculated by multiplying the amount of the error by the rate shifts.

\begin{tabular}{|llll|}
\hline The rate shifts & \multicolumn{2}{l|}{ The duration of correction (in days) } & 90 \\
\hline & 0,125 & 1 & $60 \%$ \\
\hline $\mathbf{2 \%}$ & $66 \%$ & $62 \%$ & $38 \%$ \\
\hline $\mathbf{5 \%}$ & $34 \%$ & $37,99 \%$ & $12 \%$ \\
\hline $\mathbf{3 0} \%$ & $0 \%$ & $0,01 \%$ & \\
\hline
\end{tabular}

We now study the algorithm that we described in the theoretical part :

Here, the exposed objects are of the same type, that are orders of the same nature : $X_{i}=X$, which implies that $\mathrm{P}_{\mathrm{i}}=\mathrm{P}\left(\right.$ Exposure $\left.=\mathrm{X}_{\mathrm{i}}\right)=1$.

We deduce the probability from it that the risk arises knowing that the exposure is $\mathrm{X}_{\mathrm{i}}$ :

$\mathrm{PS}_{\mathrm{i}}=\mathrm{P}\left(\right.$ Occurrence $=$ "yes" $\mid$ Exposure $\left.=\mathrm{X}_{\mathrm{i}}\right)=\mathrm{P}($ Occurrence $=$ "yes" $)=0.0728 \%$.

The algorithm consists in realizing successively the following steps :

1. calculate the distribution of severity $P_{i}=P\left(\right.$ Severity $\mid$ Occurrence $=$ "yes" and Exposure $\left.=X_{i}\right)$.

2. sample the number of losses $F_{i}$ according to binomial distribution $B\left(n b\left(X_{i}\right) ; P_{i}\right)=B(25000 ; 0,0728 \%)$. Indeed, orders are independent and each order follows a Bernoulli distribution.

3. for each incident of 1 to $F_{i}$, sample the severity according to the $\mathrm{PG}_{\mathrm{i}}$ distribution.

4. sum the severities $\mathrm{F}_{\mathrm{i}}$.

We repeat these 4 stages 10000 times by keeping the sums of the severity every time. We so obtain a distribution of total losses and we can deduct the VaR from it in 99,5 \%, [Fig. 6].

We realized tests of sensibility in the various parameters of the Bayesian network : in every set of tests, we vary the parameters of a factor by fixing the parameters of all factors.

Bayesian model has many advantages :

- It allows to take into account both quantitative factors but also qualitative factors, that do not make most of the models ;

- It allows to visualize the causal links between the variables : risk aggregation is performed by the construction of networks, thus avoiding the estimation of correlations ;

- It allows to detect factors of reduction of the risks through inference ;

- The Risk Management can use it to develop action plans and see the effectiveness of these. 
The major drawback of Bayesian networks is that they are slow to implement because they require a detailed analysis of each risk.

\section{Figures And Tables}

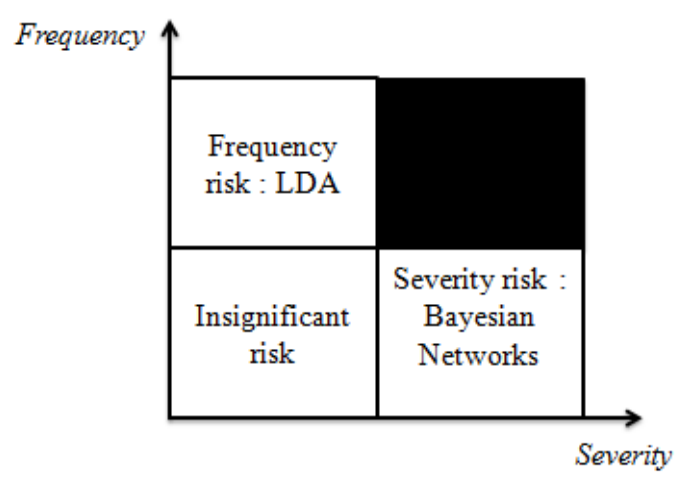

Fig 1. Matrix of Prouty for the modelling of frequency and severity risks

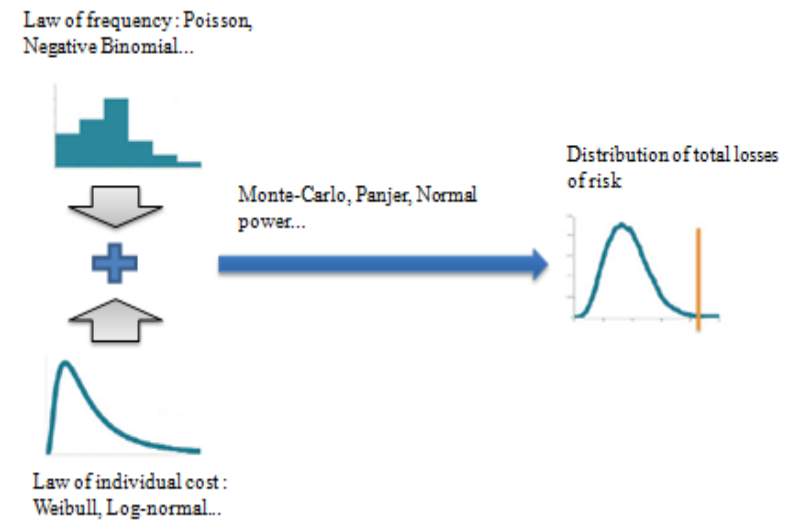

Fig 2. Construction of the distribution of the total losses

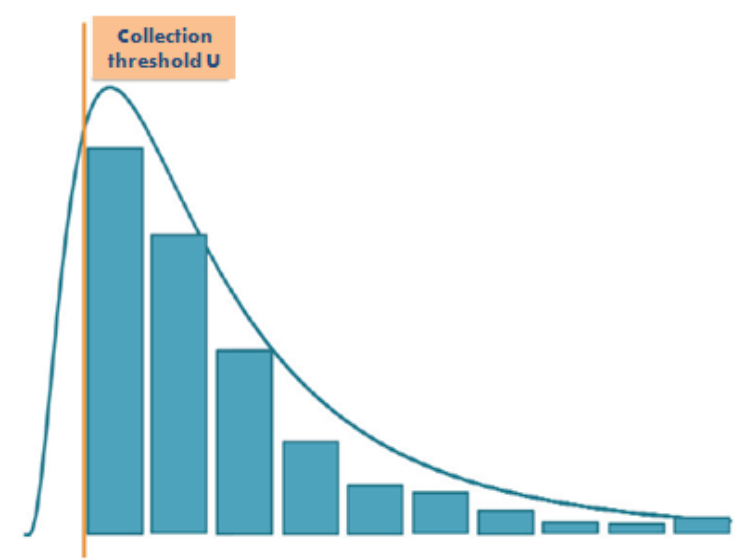

Fig 3. Cost of individual losses and the adequacy of the theoretical law adjusted To account for truncated data 


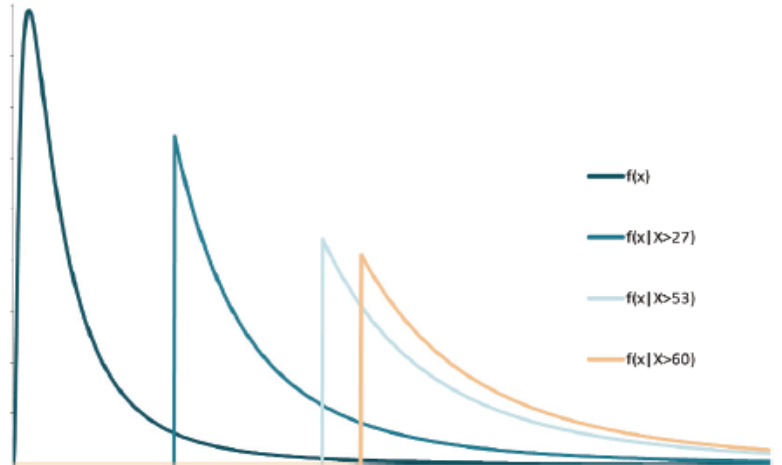

Fig 4. Deformation of a log-normal law In $(2,1)$ depending on the threshold level collections

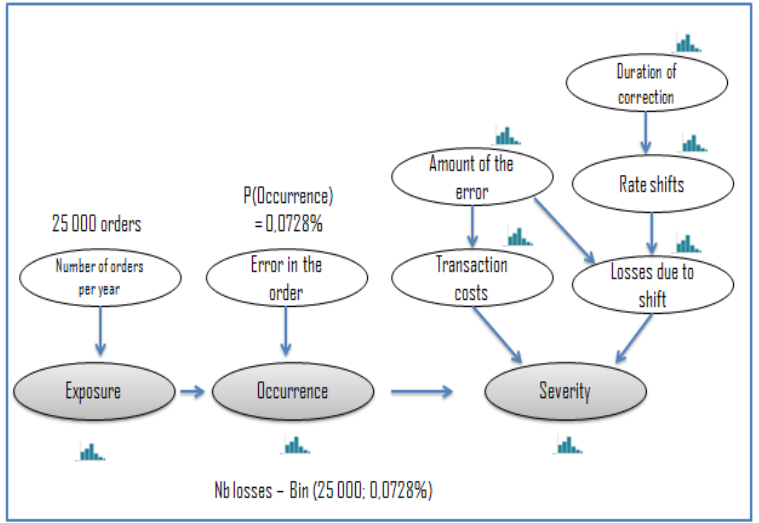

Fig 5. Application of the Bayesian model at the risk of error in the passage of orders on an insurance company

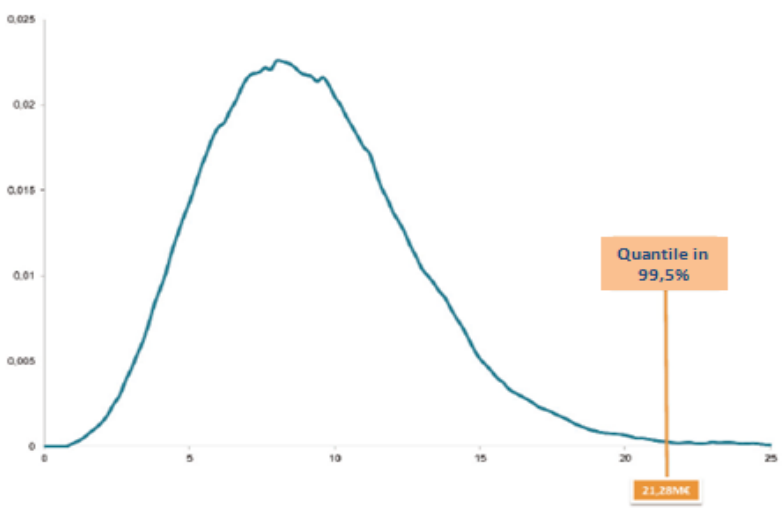

Fig 6. Distribution of total losses

\section{Conclusion}

The quantification of operational risk can not and should not be seen as an end in itself. Methodological aspects related to the use of different approaches available (LDA, Bayesian networks) and interpretation of parameters that follows, allow a more successful risk management. This is evident also in terms of both the regulator and within insurance companies.

The general idea of the LDA method is to model the loss of operational risk for a given period (eg, one year), and deduce the value-at-risk (VaR). Thus, like most models for measuring operational risk, the LDA is based on a very old actuarial approach (frequency / severity), and widely used in the field of insurance to model similar problems.

Bayesian networks are tools that have many advantages : they allow to visualize the causal links between variables, they can take into account qualitative variables, they can manage risks by identifying variables levers. This model will be useful to Risk Management to control the risks. The major drawback of Bayesian networks is that they are slow to implement. Another faster solution to model the risks of severity would be the law of Extremes. 


\section{References}

[1] Alexander, C., (2003). Operational Risk : Regulation, Analysis and Management. Ed. FT Prentice Hall, 336 p.

[2] Arzac, R. E., (1976). Profits and Safety in the Theory of the firm under Price Uncertainty. International Economic Preview, 17, 163171 .

[3] Bee, M., (2006). The Advanced Measurement Approach to Operational Risk. London, Ed. E. Davis, Risk Books, 350 p.

[4] Bernouilli, D. (1954). Exposition of New Theory of the Measurement of Risk. Econometrica, 22, $123-136$.

[5] Chavez-Demoulin, V., P. Embrechts and, J. Nešlehová, (2006). Quantitative Models for Operational Risk: Extremes, Dependence and Aggregation. Journal of Banking and Finance, 30, 10, 2635-2658.

[6] Chernobai, A., C. Menn, S. Trûck and S. T. Rachev, (2005a). A Note on the Estimation of the Frequency and Severity Distribution of Operational Losses. Mathematical Scientist 30, 2, 87-97.

[7] Coles S., Powell E., (1996). Bayesian methods in extreme value modelling: a review and new developments. Internat. Statist. Rev. 64, 119-36.

[8] Condamin, L., Louisot, J.-P. ; Naîm, P., (2007). Risk Quantification. Ed. Wiley, 286 p.

[9] El Sayyad, G. M. (1973). Bayesian and Classical analysis of Poisson Regression. Journal of the Royal Statistical Society, Series B, $35,445-451$

[10] Frachot, A., O., Moudoulaud, T., Roncalli, (2004). Loss Distribution Approach in Practice. The Basel Handbook : A Guide for Financial Practioners. Micheal Ong, Risk Books. Electronic copy available at: http://ssrn.com/abstract=1032592.

[11] Naïm P. ; Wuillemin P.-H., Leray P., Pourret O., Becker A., (2007). Réseaux Bayésiens. Ed. Eyrolles, Collection Algorithmes, 424 p.

[12] Partrat C., Besson J.-L., (2004), Assurance non-vie, Modélisation et Simulation. Economica, 820 p. 